\title{
A uniqueness result for the Sine-Gordon breather
}

\section{Rainer Mandel ${ }^{1}$}

Received: 2 February 2021 / Accepted: 24 February 2021 / Published online: 12 March 2021

(C) The Author(s) 2021

\section{Abstract}

In this note we prove that the sine-Gordon breather is the only quasimonochromatic breather in the context of nonlinear wave equations in $\mathbb{R}^{N}$.

Mathematics Subject Classification 35J05 $\cdot 35 \mathrm{~J} 60 \cdot 35 \mathrm{~L} 05 \cdot 35 \mathrm{~L} 70$

\section{Introduction}

Breathers are time-periodic and spatially localized patterns that describe the propagation of waves. The most impressive solution of this kind is the so-called sine-Gordon breather for the 1D sine-Gordon equation

$$
\partial_{t t} u-\partial_{x x} u+\sin (u)=0 \quad \text { in } \mathbb{R} \times \mathbb{R} .
$$

It is given by the explicit formula

$$
u^{*}(x, t)=4 \arctan \left(\frac{m \sin (\omega t)}{\omega \cosh (m x)}\right) \quad \text { for }(x, t) \in \mathbb{R} \times \mathbb{R},
$$

where the parameters $m, \omega>0$ satisfy $m^{2}+\omega^{2}=1$. It is natural to ask if other real-valued breather solutions exist. We shall address this question in the broader context of more general nonlinear wave equations of the form

$$
\partial_{t t} u-\Delta u=g(u) \text { in } \mathbb{R}^{N} \times \mathbb{R},
$$

where the space dimension $N \in \mathbb{N}$ and the nonlinearity $g: \mathbb{R} \rightarrow \mathbb{R}$ are arbitrary.

The existence of radially symmetric breather solutions for the cubic Klein-Gordon equation $g(z)=-m^{2} z+z^{3}, m>0$ in three spatial dimensions was established in [13]. These real-valued solutions are only weakly localized in the sense that they satisfy $u(\cdot, t) \in$ $L^{q}\left(\mathbb{R}^{N}\right)$ for some $q \in(2, \infty)$ but $u(\cdot, t) \notin L^{2}\left(\mathbb{R}^{N}\right)$. In [10] infinitely many weakly localized

This article is part of the section "Theory ofPDEs" edited by Eduardo Teixeira.

Rainer Mandel

rainer.mandel@kit.edu

1 Karlsruhe Institute of Technology, Institute for Analysis, Englerstraße 2, 76131 Karlsruhe, Germany 
breathers were found for nonlinearities $Q(x)|u|^{p-2} u$ where $Q$ lies in a suitable Lebesgue space and $p>2$ is chosen suitably depending on $Q$ as well as the space dimension $N \geq 2$. Up to now, nothing is known about the existence of strongly localized breathers of Eq. (2) satisfying $u(\cdot, t) \in L^{2}\left(\mathbb{R}^{N}\right)$ for almost all $t \in \mathbb{R}$ and $N \geq 2$, see however [11] for a an existence result for semilinear curl-curl equations for $N=3$. In the case $N=1$ strongly localized breather solutions different from the sine-Gordon breather have been found for nonlinear wave equations of the form

$$
s(x) \partial_{t t} u-u_{x x}+q(x) u=f(x, u) \quad(x \in \mathbb{R})
$$

where the coefficient functions $s, q$ are discontinuous and periodic, see [7, Theorem 1.3] and [1, Theorem 1.1]. Given the discontinuity of $s, q$ it must be expected that these breathers are not twice continuously differentiable. To sum up, the existence of smooth and strongly localized breather solutions of Eq. (2) different from the sine-Gordon breather is not known. Still for $N=1$ there are nonexistence results by Denzler [4] and Kowalczyk et al. [9] dealing with small perturbations of the sine-Gordon equation respectively small odd breathers (not covering the even sine-Gordon breather). We are not aware of any other mathematically rigorous existence or nonexistence results for Eq. (2).

One of the main obstructions for the construction of localized breathers is polychromaticity. Indeed, plugging in an ansatz of the form $u(x, t)=\sum_{k \in \mathbb{Z}} u_{k}(x) e^{i k t}$ with $u_{k}=\overline{u_{-k}}$ one ends up with infinitely many equations of nonlinear Helmholtz type that typically do not possess strongly localized solutions, see for instance [8, Theorem 1a]. For this reason the solutions obtained in $[10,13]$ are only weakly localized. On the other hand, a purely monochromatic ansatz like $u(x, t)=\sin (\omega t) p(x)$ cannot be successful either provided that $g$ is not a linear function. In view of the formula (1) for the sine-Gordon breather we investigate whether quasimonochromatic breathers exist.

Definition 1 We call the function $u: \mathbb{R}^{N} \times \mathbb{R} \rightarrow \mathbb{R}$ a quasimonochromatic breather if

$$
u(x, t)=F(\sin (\omega t) p(x)) \quad\left(x \in \mathbb{R}^{N}, t \in \mathbb{R}\right)
$$

for some $\omega \in \mathbb{R} \backslash\{0\}$ and nontrivial functions $F \in C^{2}(\mathbb{R}), p \in C^{2}\left(\mathbb{R}^{N}\right)$ such that $F(0)=0$ and $p(x) \rightarrow 0$ as $|x| \rightarrow \infty$.

We show that in one spatial dimension the sine-Gordon breather is, up to translation and dilation, the only one for (2) and that no such breathers exist in higher dimensions as long as $g$ does not act like a linear function. In fact, to rule out $L^{\infty}$-small solutions of linear wave equations, we assume that $g: \mathbb{R} \rightarrow \mathbb{R}$ is not a linear function near zero, i.e., that there is a nontrivial interval $I \subset \mathbb{R}$ containing 0 with the property that there is no $\beta \in \mathbb{R}$ such that $g(z)=\beta z$ for all $z \in I$.

\section{Theorem 1 Assume $N \in \mathbb{N}$ and that $g: \mathbb{R} \rightarrow \mathbb{R}$ is not a linear function near zero.}

(i) In the case $N \geq 2$ there is no quasimonochromatic breather solution of (2).

(ii) In the case $N=1$ each quasimonochromatic breather solution of (2) is of the form $u(x, t)=\kappa u^{*}\left(x-x_{0}, t\right)$ for $x_{0} \in \mathbb{R}, m, \omega, \kappa \in \mathbb{R} \backslash\{0\}$ and $u^{*}$ as in (1). The nonlinearity then satisfies $g(z)=-\left(m^{2}+\omega^{2}\right) \kappa \sin \left(\kappa^{-1} z\right)$ whenever $|z|<2 \pi|\kappa|$.

We stress that our result holds regardless of any smoothness assumption on $g$ nor any kind of growth condition at 0 or infinity. Moreover, our considerations are not limited to small 
perturbations of $u^{*}$ or small breathers in whatever sense. Following the proof of Theorem 1 one also finds that quasimonochromatic breathers of wave equations on any open set $\Omega \subsetneq \mathbb{R}^{N}$ with homogeneous Dirichlet conditions

$$
\partial_{t t} u-\Delta u=g(u) \quad \text { in } \Omega \times \mathbb{R}, \quad u=0 \text { on } \partial \Omega \times \mathbb{R}
$$

with profile functions $p \in C^{2}(\bar{\Omega})$ do not exist either (even if $N=1$ ) provided that $g$ is not a linear function near zero. We will comment on this fact at the end of this paper. As a consequence, we find that Rabinowitz' $C^{2}([0,1] \times \mathbb{R})$-solutions of the $1 \mathrm{D}$ wave equation from [12, Theorem 1.6] are not of quasimonochromatic type. This might be true as well for the solutions from [2,3], but here our argument does not apply in a direct way since the solutions are not known to be twice continuously differentiable up to the boundary.

For completeness we briefly comment on the linear case $g(z)=\beta z, \beta \in \mathbb{R}$. Then the profile function $p$ of any given quasimonochromatic breather of (2) satisfies the linear elliptic PDE $-\Delta p-\left(\omega^{2}+\beta\right) p=0$ in $\mathbb{R}^{N}$.

For $\beta<-\omega^{2}$ there are positive, radially symmetric and exponentially decaying solutions $p$, see [5, Theorem 2]. In the case $\beta>-\omega^{2}, N \geq 2$ one can find radial as well as nonradial solutions of the associated Helmholtz equation all of which have infinitely many nodal domains and satisfy $|p(x)|+|\nabla p(x)| \gtrsim|x|^{\frac{1-N}{2}}$ in a suitable integrated sense, see [14, Theorem 1] respectively [8, Theorem 1a]. For $\beta>-\omega^{2}, N=1$ all solutions are linear combinations of sin and cos so that breather solutions do not exist. So we see that the picture is already quite complete in the case of linear wave equations.

\section{Proof of Theorem 1}

In the following let $u(x, t)=F(\sin (\omega t) p(x))$ be a solution of (2) with $g$ as in the Theorem. Plugging in this ansatz we get for all $x \in \mathbb{R}^{N}$ such that $p(x) \neq 0$,

$$
\begin{aligned}
\partial_{t t} u(x, t) & =-\omega^{2} \sin (\omega t) p(x) F^{\prime}(\sin (\omega t) p(x))+\omega^{2} \cos (\omega t)^{2} p(x)^{2} F^{\prime \prime}(\sin (\omega t) p(x)) \\
& =-\omega^{2} z F^{\prime}(z)+\omega^{2}\left(p(x)^{2}-z^{2}\right) F^{\prime \prime}(z), \\
\Delta u(x, t) & =\sin (\omega t) \Delta p(x) F^{\prime}(\sin (\omega t) p(x))+\sin (\omega t)^{2}|\nabla p(x)|^{2} F^{\prime \prime}(\sin (\omega t) p(x)) \\
& =\frac{\Delta p(x)}{p(x)} z F^{\prime}(z)+\frac{|\nabla p(x)|^{2}}{p(x)^{2}} z^{2} F^{\prime \prime}(z),
\end{aligned}
$$

where $z=\sin (\omega t) p(x) \in\left[-\|p\|_{\infty},+\|p\|_{\infty}\right]$. This and (2) imply for $x \in \mathbb{R}^{N}, z \in \mathbb{R}$ such that $p(x) \neq 0, z \in\left[-\|p\|_{\infty},+\|p\|_{\infty}\right]$

$$
g(F(z))+\omega^{2} z F^{\prime}(z)+\omega^{2} z^{2} F^{\prime \prime}(z)=p(x)^{2} \omega^{2} F^{\prime \prime}(z)-\frac{\Delta p(x)}{p(x)} z F^{\prime}(z)-\frac{|\nabla p(x)|^{2}}{p(x)^{2}} z^{2} F^{\prime \prime}(z) .
$$

If $F$ was linear on $\left[-\|p\|_{\infty},+\|p\|_{\infty}\right]$, then $g$ would have to be linear on the nontrivial interval $I:=\left\{F(z):|z| \leq\|p\|_{\infty}\right\}$ as well. Since the latter is not the case by assumption, we know that $z \mapsto z^{2} F^{\prime \prime}(z)$ does not vanish identically on that interval. Multiplying (4) with $p(x)$ and choosing $z$ according to $z^{2} F^{\prime \prime}(z) \neq 0$ we find that $p$ does not change sign. Indeed, if $p\left(x^{*}\right) \neq 0$ and $R>0$ is the smallest radius such that $p$ has a fixed sign in the open ball $B_{R}\left(x^{*}\right)$, then Hopf's Lemma [6, Lemma 3.4] implies $|\nabla p|>0$ on $\partial B_{R}\left(x^{*}\right)$. But then (4) 
implies that $\Delta p$ is unbounded on $\partial B_{R}\left(x^{*}\right)$, which contradicts $p \in C^{2}\left(\mathbb{R}^{N}\right)$. Hence, $p$ does not change sign and we will without loss of generality assume that $p$ is positive. So (4) holds for all $x \in \mathbb{R}^{N}$ and all $z \in\left[-\|p\|_{\infty},\|p\|_{\infty}\right]$ and standard elliptic regularity theory gives $p \in C^{\infty}\left(\mathbb{R}^{N}\right)$.

Differentiating (4) with respect to $x_{i}$ we get

$$
\partial_{i}\left(p(x)^{2}\right) \omega^{2} F^{\prime \prime}(z)-\partial_{i}\left(\frac{\Delta p(x)}{p(x)}\right) z F^{\prime}(z)-\partial_{i}\left(\frac{|\nabla p(x)|^{2}}{p(x)^{2}}\right) z^{2} F^{\prime \prime}(z)=0 .
$$

Since $p^{2}$ is non-constant, we infer that $F$ satisfies an ODE of the form

$$
F^{\prime \prime}(z)=\frac{-\mu_{2} z}{\omega^{2}+\mu_{1} z^{2}} F^{\prime}(z) \quad\left(|z| \leq\|p\|_{\infty}, \mu_{1} \in \mathbb{R}, \mu_{2} \in \mathbb{R} \backslash\{0\}\right) .
$$

Here, $\mu_{2} \neq 0$ is due to the fact that $F$ is not a linear function. Each nontrivial solution of such an ODE satisfies $F^{\prime}(z) \neq 0$ for almost all $z \in\left[-\|p\|_{\infty},\|p\|_{\infty}\right]$. Combining (5) and (6) we thus infer

$$
-\partial_{i}\left(p(x)^{2}\right) \frac{\mu_{2} \omega^{2} z}{\omega^{2}+\mu_{1} z^{2}}-\partial_{i}\left(\frac{\Delta p(x)}{p(x)}\right) z+\partial_{i}\left(\frac{|\nabla p(x)|^{2}}{p(x)^{2}}\right) \frac{\mu_{2} z^{3}}{\omega^{2}+\mu_{1} z^{2}}=0 .
$$

Since (6) holds for all $i \in\{1, \ldots, N\}$ and $z \in\left[-\|p\|_{\infty},\|p\|_{\infty}\right]$, we get

$$
\begin{aligned}
-\mu_{1} \partial_{i}\left(\frac{\Delta p(x)}{p(x)}\right)+\mu_{2} \partial_{i}\left(\frac{|\nabla p(x)|^{2}}{p(x)^{2}}\right) & =0, \\
-\mu_{2} \partial_{i}\left(p(x)^{2}\right)-\partial_{i}\left(\frac{\Delta p(x)}{p(x)}\right) & =0 .
\end{aligned}
$$

Since $\mu_{2} \neq 0$ we can find $\lambda_{1}, \lambda_{2} \in \mathbb{R}$ such that

$$
-\mu_{1} \frac{\Delta p}{p}+\mu_{2} \frac{|\nabla p|^{2}}{p^{2}}=-\lambda_{2} \mu_{1}+\lambda_{1} \mu_{2}, \quad-\mu_{2} p^{2}-\frac{\Delta p}{p}=-\lambda_{2} .
$$

This implies

$$
|\nabla p|^{2}=\lambda_{1} p^{2}-\mu_{1} p^{4}, \quad-\Delta p+\lambda_{2} p=\mu_{2} p^{3} .
$$

We now use (7) and the positivity of $p$ to show that $p$ is radially symmetric about its maximum point $x_{0} \in \mathbb{R}^{N}$. We concentrate on the case $N \geq 2$ since the claim for $N=1$ follows from the fact that $x \mapsto u\left(x_{0}+x\right)$ and $x \mapsto u\left(x_{0}-x\right)$ solve the same initial value problem. Since $p$ vanishes at infinity, we must have $\lambda_{1} \geq 0$ and, since $p$ does not change sign, $\lambda_{2} \geq 0$, see [14, Theorem 1]. Moreover, $p$ attains its maximum at some point $x_{0} \in \mathbb{R}^{N}$ with $p\left(x_{0}\right)>0,\left|\nabla p\left(x_{0}\right)\right|=0, \Delta p\left(x_{0}\right) \leq 0$. This and (7) implies $\lambda_{1}, \mu_{1}>0$ as well as $\mu_{2} \geq 0$. So we know that (7) holds for

$$
\lambda_{1}, \mu_{1}>0, \quad \lambda_{2}, \mu_{2} \geq 0 .
$$

In the case $\lambda_{2}>0$ Theorem 2 from [5] implies the radial symmetry about $x_{0}$, so we are left with the case $\lambda_{2}=0$. 
So let use assume $\lambda_{2}=0$. Liouville's Theorem implies that $\mu_{2}=0$ is impossible, so we have $\mu_{2}>0$ in this case. Define $\alpha:=1-\frac{\mu_{2}}{\mu_{1}} \in(-\infty, 1)$. In the case $\alpha \in(0,1)$ the function $\psi(x):=p(x)^{\alpha}$ satisfies

$$
-\Delta \psi=-\alpha(\Delta p) p^{\alpha-1}-\alpha(\alpha-1)|\nabla p|^{2} p^{\alpha-2} \stackrel{(7)}{=} \alpha(1-\alpha) \lambda_{1} \psi .
$$

In view of $\alpha(1-\alpha) \lambda_{1}>0$ Theorem 1 from [14] implies that $\psi$ has infinitely many nodal domains, which contradicts the positivity of $\psi$. So this case cannot occur. In the case $\alpha \in(-\infty, 0)$ radial symmetry about $x_{0}$ follows once more from [5, Theorem 2], so it remains to discuss the case $\alpha=0$, i.e., $\mu_{1}=\mu_{2}$. Then $\psi(x):=\log (p(x))$ satisfies

$$
-\Delta \psi=-(\Delta p) p^{-1}+|\nabla p|^{2} p^{-2} \stackrel{(7)}{=} \lambda_{1} \psi
$$

and we find as above that $\psi$ has to change sign infinitely many times, which is a contradiction. So we have shown that $p$ is radially symmetric about $x_{0}$ also in the case $\lambda_{2}=0$.

So we have

$$
p(x)=p_{0}\left(\left|x-x_{0}\right|\right) \quad \text { where } p_{0}^{\prime}(r)^{2}=\lambda_{1} p_{0}(r)^{2}-\mu_{1} p_{0}(r)^{4}, \quad p_{0}^{\prime}(0)=0 .
$$

Solving this ODE gives

$$
p_{0}(r)=\frac{A}{\cosh (m r)} \quad \text { where } \lambda_{1}=m^{2}, \mu_{1}=m^{2} A^{-2}
$$

for some $A>0, m \neq 0$. So $-\Delta p+\lambda_{2} p=\mu_{2} p^{3}$ can only hold for $N=1$ as well as $\lambda_{2}=m^{2}$, $\mu_{2}=2 m^{2} A^{-2}$. Plugging these values into (6) and solving the ODE we get from $F(0)=0, F \not \equiv 0$

$$
F(z)=4 \kappa \arctan \left(\frac{m z}{A \omega}\right) \quad \text { for some } \kappa \in \mathbb{R} \backslash\{0\} .
$$

This implies that the breather solution is given by

$$
u(x, t)=F(\sin (\omega t) p(x))=F\left(\sin (\omega t) p_{0}\left(\left|x-x_{0}\right|\right)\right)=\kappa u^{*}\left(x-x_{0}, t\right)
$$

for $u^{*}$ as in (1). So have proved the nonexistence of such breathers for $N \geq 2$ from claim (i) and the uniqueness statement from claim (ii).

To see that this solution formula determines the nonlinearity $g$, we combine (6) and (7) to get

$$
p(x)^{2} \omega^{2} F^{\prime \prime}(z)-\frac{\Delta p(x)}{p(x)} z F^{\prime}(z)-\frac{|\nabla p(x)|^{2}}{p(x)^{2}} z^{2} F^{\prime \prime}(z)=\frac{m^{2}\left(m^{2} z^{2}-A^{2} \omega^{2}\right)}{m^{2} z^{2}+A^{2} \omega^{2}} F^{\prime}(z) z .
$$

So (4) implies

$$
\begin{aligned}
g(F(z)) & =-\omega^{2} z F^{\prime}(z)-\omega^{2} z^{2} F^{\prime \prime}(z)+\frac{m^{2}\left(m^{2} z^{2}-A^{2} \omega^{2}\right)}{m^{2} z^{2}+A^{2} \omega^{2}} F^{\prime}(z) z \\
& =\frac{\left(m^{2}+\omega^{2}\right)\left(m^{2} z^{2}-A^{2} \omega^{2}\right)}{m^{2} z^{2}+A^{2} \omega^{2}} z F^{\prime}(z) \\
& =\frac{4 A m \kappa \omega\left(m^{2}+\omega^{2}\right)\left(m^{2} z^{2}-A^{2} \omega^{2}\right) z}{\left(m^{2} z^{2}+A^{2} \omega^{2}\right)^{2}} .
\end{aligned}
$$


Plugging in $z=\frac{A \omega}{m} \tan \left(\frac{y}{4 \kappa}\right)$ for $|y|<2 \pi|\kappa|$ we get $F(z)=y$ and hence

$$
\begin{aligned}
g(y) & =\frac{4 A^{2} \omega^{2} \kappa\left(m^{2}+\omega^{2}\right)\left(A^{2} \omega^{2} \tan \left(\frac{y}{4 \kappa}\right)^{2}-A^{2} \omega^{2}\right) \tan \left(\frac{y}{4 \kappa}\right)}{\left(A^{2} \omega^{2} \tan \left(\frac{y}{4 \kappa}\right)^{2}+A^{2} \omega^{2}\right)^{2}} \\
& =\frac{4 \kappa\left(m^{2}+\omega^{2}\right)\left(\tan \left(\frac{y}{4 \kappa}\right)^{2}-1\right) \tan \left(\frac{y}{4 \kappa}\right)}{\left(\tan \left(\frac{y}{4 \kappa}\right)^{2}+1\right)^{2}} \\
& =4 \kappa\left(m^{2}+\omega^{2}\right)\left(\sin \left(\frac{y}{4 \kappa}\right)^{2}-\cos \left(\frac{y}{4 \kappa}\right)^{2}\right) \sin \left(\frac{y}{4 \kappa}\right) \cos \left(\frac{y}{4 \kappa}\right) \\
& =-2 \kappa\left(m^{2}+\omega^{2}\right) \cos \left(\frac{y}{2 \kappa}\right) \sin \left(\frac{y}{2 \kappa}\right) \\
& =-\kappa\left(m^{2}+\omega^{2}\right) \sin \left(\frac{y}{\kappa}\right) .
\end{aligned}
$$

\section{Remark 1}

(i) We explain why nonlinear quasimonochromatic breathers of (3) with profile functions $p \in C^{2}(\bar{\Omega})$ do not exist on open sets $\Omega \subsetneq \mathbb{R}^{N}$. The arguments presented above reveal that any such breather is given by functions $F, p$ as in Definition 1 such that for all $x \in \Omega, p(x) \neq 0,|z| \leq\|p\|_{\infty}$ we have as in (4)

$$
g(F(z))+\omega^{2} z F^{\prime}(z)+\omega^{2} z^{2} F^{\prime \prime}(z)=p(x)^{2} \omega^{2} F^{\prime \prime}(z)-\frac{\Delta p(x)}{p(x)} z F^{\prime}(z)-\frac{|\nabla p(x)|^{2}}{p(x)^{2}} z^{2} F^{\prime \prime}(z) .
$$

Now fix $z \in\left(-\|p\|_{\infty},\|p\|_{\infty}\right)$ such that $z^{2} F^{\prime \prime}(z) \neq 0$ and choose $x^{*} \in \Omega$ such that $p\left(x^{*}\right) \neq 0$. Let $R>0$ be largest possible such that $|p|$ is positive in the open ball $B_{R}\left(x^{*}\right) \subset \Omega$. By the homogeneous Dirichlet boundary condition, we know $R \leq \operatorname{dist}\left(x^{*}, \partial \Omega\right)<\infty$ and that $p$ vanishes on $\partial B_{R}\left(x^{*}\right)$. So the same argument as in the above proof (Hopf's Lemma) shows that $|\Delta p|$ is unbounded on $B_{R}\left(x^{*}\right)$, a contradiction. As a consequence, such a profile function cannot exist and we obtain the nonexistence of quasimonochromatic breathers for (3).

(ii) In our proof we did not use the assumption $p(x) \rightarrow 0$ as $|x| \rightarrow \infty$ when we proved that $|p|$ is positive. As a consequence, each profile function $p$ of a solution $u(x, t)=F(\sin (\omega t) p(x))$ of (2) has a fixed sign regardless of its behaviour at infinity. Similarly, (7) holds without this hypothesis. So we conclude that any profile function $p \in C^{2}\left(\mathbb{R}^{N}\right)$ of a quasimonochromatic breather is a positive solution of (7) provided that the nonlinearity $g$ is not a linear function on the interval $\left\{F(z):|z| \leq\|p\|_{\infty}\right\}$. Notice also that the assumption $F(0)=0$ is not used either.

(iii) Our notion of a quasimonochromatic breather does not allow for the solutions $u(x, t)=u^{*}\left(x_{1}, t\right)\left(x \in \mathbb{R}^{N}\right)$, which are localized only with respect to one spatial direction.

Accordingly, our nonexistence result for $N \geq 2$ is false under the weaker requirement 


$$
\sup _{x^{\prime} \in \mathbb{R}^{N-1}}\left|p\left(x_{1}, x^{\prime}\right)\right| \rightarrow 0 \quad \text { as } x_{1} \rightarrow \infty .
$$

One may conjecture that the solutions $u(x, t)=u^{*}(x \cdot \theta, t)$ for $\theta \in S^{N-1} \subset \mathbb{R}^{N}$ are the only quasimonochromatic breathers that are localized in some spatial direction. This open problem bears some similarity to the Gibbon's Conjecture or de Giorgi Conjecture about the classification of monotone solutions of the AllenCahn equation $\Delta u+u=u^{3}$ in $\mathbb{R}^{N}$ that we recast in our setting below.

Conjecture 1 Let $N \in \mathbb{N}, N \geq 2$ and let $p \in C^{2}\left(\mathbb{R}^{N}\right)$ be a solution of (7) for some $\lambda_{1}, \lambda_{2}, \mu_{2}, \mu_{2} \in \mathbb{R}$ that satisfies (8). Then there are $\gamma, m, z \in \mathbb{R}$ such that

$$
p(x)=\frac{\gamma}{\cosh \left(m\left(x_{1}-z\right)\right)} .
$$

Conjecture 2 Let $N \in \mathbb{N}, N \geq 2$ and let $p \in C^{2}\left(\mathbb{R}^{N}\right)$ be a solution of (7) for some $\lambda_{1}, \lambda_{2}, \mu_{2}, \mu_{2} \in \mathbb{R}$ that satisfies $\partial_{1} p(x) x_{1}<0$ for all $x \in \mathbb{R}^{N}$ such that $x_{1} \neq 0$. Then there are $\gamma, m>0$ such that

$$
p(x)=\frac{\gamma}{\cosh \left(m x_{1}\right)} .
$$

Acknowledgements The author thanks the referee for indicating a connection to de Giorgi's Conjecture. Funded by the Deutsche Forschungsgemeinschaft (DFG, German Research Foundation) - Project-ID 258734477 - SFB 1173.

Open Access This article is licensed under a Creative Commons Attribution 4.0 International License, which permits use, sharing, adaptation, distribution and reproduction in any medium or format, as long as you give appropriate credit to the original author(s) and the source, provide a link to the Creative Commons licence, and indicate if changes were made. The images or other third party material in this article are included in the article's Creative Commons licence, unless indicated otherwise in a credit line to the material. If material is not included in the article's Creative Commons licence and your intended use is not permitted by statutory regulation or exceeds the permitted use, you will need to obtain permission directly from the copyright holder. To view a copy of this licence, visit http://creativecommons.org/licenses/by/4.0/.

Funding Open Access funding enabled and organized by Projekt DEAL.

\section{References}

1. Blank, C., Chirilus-Bruckner, M., Lescarret, V., Schneider, G.: Breather solutions in periodic media. Comm. Math. Phys. 302(3), 815-841 (2011)

2. Brézis, H., Coron, J.-M., Nirenberg, L.: Free vibrations for a nonlinear wave equation and a theorem of P. Rabinowitz. Commun. Pure Appl. Math. 33(5), 667-684 (1980)

3. Coron, J.-M.: Periodic solutions of a nonlinear wave equation without assumption of monotonicity. Math. Ann. 262(2), 273-285 (1983)

4. Denzler, J.: Nonpersistence of breather families for the perturbed sine Gordon equation. Comm. Math. Phys. 158(2), 397-430 (1993) 
5. Gidas, B., Ni, Wei Ming, Nirenberg, L.: Symmetry of positive solutions of nonlinear elliptic equations in $\mathbf{R}^{n}$. In: Mathematical analysis and applications, Part A, volume 7 ofAdv. in Math. Suppl. Stud., pages 369-402. Academic Press, New York-London, (1981)

6. Gilbarg, D., Trudinger, N.S.: Elliptic partial differential equations of second order. Classics in Mathematics. Springer-Verlag, Berlin (2001). (Reprint of the 1998 edition)

7. Hirsch, A., Reichel, W.: Real-valued, time-periodic localized weak solutions for a semilinear wave equation with periodic potentials. Nonlinearity 32(4), 1408-1439 (2019)

8. Kato, T.: Growth properties of solutions of the reduced wave equation with a variable coefficient. Comm. Pure Appl. Math. 12, 403-425 (1959)

9. Kowalczyk, M., Martel, Y., Muñoz, C.: Nonexistence of small, odd breathers for a class of nonlinear wave equations. Lett. Math. Phys. 107(5), 921-931 (2017)

10. Mandel, R., Scheider, D.: Variational methods for breather solutions of nonlinear wave equations. Nonlinearity. (2021). arXiv:2009.02028

11. Plum, M., Reichel, W.: A breather construction for a semilinear curl-curl wave equation with radially symmetric coefficients. J. Elliptic Parabol. Equ. 2(1-2), 371-387 (2016)

12. Rabinowitz, P.H.: Free vibrations for a semilinear wave equation. Commun. Pure Appl. Math. 31(1), 31-68 (1978)

13. Scheider, D.: Breather solutions of the cubic Klein-Gordon equation. Nonlinearity 33(12), 7140-7166 (2020)

14. Swanson, C.A.: Semilinear second-order elliptic oscillation. Canad. Math. Bull. 22(2), 139-157 (1979) 